\section{Cahiers de Narratologie}

Analyse et théorie narratives

29 | 2015

Street Art 1

\title{
Le récit contradictoire et son linguistic turn
}

\section{Marie-Albane Watine}

\section{OpenEdition}

\section{Journals}

Electronic version

URL: http://journals.openedition.org/narratologie/7372

DOI: 10.4000/narratologie.7372

ISSN: 1765-307X

\section{Publisher}

LIRCES

\section{Electronic reference}

Marie-Albane Watine, "Le récit contradictoire et son linguistic turn », Cahiers de Narratologie [Online] 29 | 2015, Online since 04 December 2015, connection on 19 April 2019. URL : http:// journals.openedition.org/narratologie/7372; DOI : 10.4000/narratologie.7372

This text was automatically generated on 19 April 2019

Article L.111-1 du Code de la propriété intellectuelle. 


\title{
Le récit contradictoire et son linguistic turn
}

\author{
Marie-Albane Watine
}

1 Nous nous proposons dans cette étude de mettre en évidence les caractéristiques narratologiques, contextuelles et linguistiques de ce que nous appellerons les "récits contradictoires ». Nous désignons par là des récits qui font, en quelque façon, entorse au principe de non-contradiction tel qu'il est défini chez Aristote : « Il est impossible qu'un même attribut appartienne et n'appartienne pas en même temps et sous le même rapport à une même chose ${ }^{1}$ ».

2 La contradiction narrative n'est pas un terrain inexploré dans l'étude du récit, loin s'en faut : elle entre notamment dans les composantes de ce que Brian McHale a appelé les «fictions postmodernes ${ }^{2}$ ». Elle présente aussi une parenté avec la notion de « discohérence », créée en 1973 par Jean Ricardou pour caractériser certains récits du " Nouveau Nouveau Roman », qui « met[tent] en scène l'assemblage impossible d'un Pluriel diégétique $e^{3}$ "- mais la mise en œuvre de cette notion cette fois-ci étroite nous paraît surtout opératoire pour les textes de cette période. La contradiction peut enfin entrer dans les caractéristiques plus larges de ce que Francis Berthelot appelle les «transfictions », qui, placées à l'interface entre narratologie thématique et narratologie discursive, bouleversent « l'ordre du monde actuel » en mêlant réel et imaginaire, et/ou «l'ordre du récit» en contestant les formes narratives couramment utilisées depuis le XIXe siècle ${ }^{4}$ : ainsi les textes de Buzzati, Auster, Queneau, Pynchon ou encore Lovecraft, Dick ou Matheson que Berthelot recense dans son anthologie.

3 Nous souhaitons pour notre part nous placer d'un point de vue logique et linguistique pour borner l'exploration de ces transgressions postmodernes à la seule expression de la contradiction. Un récit est contradictoire, non parce qu'il évoque des faits qui n'appartiennent pas à la réalité de notre monde (comme c'est le cas dans le merveilleux ou le fantastique), mais parce qu'il affirme ou oblige à inférer, en même temps, deux propositions contradictoires $P$ et non- $P$.

4 Quels sont les propriétés de ce type de récit? Comment expliquer leur fortune à partir des années 1960 - la contradiction serait-elle un objet spécifiquement postmoderne? 
Dans quelle mesure et par quels moyens linguistiques la contradiction bouleverse-t-elle les cadres du récit, voire du discours dans son ensemble? Quelle est l'influence des données contextuelles sur les formes adoptées par ce type de récit?

$5 \quad$ Nous nous appuierons dans un premier temps sur un texte d'Aragon pour mettre au jour le fonctionnement et les enjeux d'un récit contradictoire, avant de montrer quelle convergence de données historiques et culturelles président à la multiplication des récits contradictoires dans les décennies concernées. Nous confronterons enfin le récit d'Aragon à un court texte d'Allais (1890), pour montrer que leur fonctionnement linguistique respectif autorise à en distinguer deux modèles formels, qui témoignent du fait que le récit contradictoire est entré dans l'ère du « tournant linguistique ».

\section{Un récit contradictoire: « Histoire d'Angus et de Jessica »}

6 En 1967, Aragon publie Blanche ou l'oubli5. Ce roman, qui prend place au sein de la mise en crise du roman des années 1950 et 1960, cultive l'incertitude de l'origine narrative, la problématisation de la notion de personnage et la remise en question de la linéarité. Autant dire qu'un tel roman, de plus particulièrement touffu et foisonnant, est difficile à présenter dans son ensemble ; mais le récit contradictoire qui nous intéresse au premier chef forme surtout la matière d'un chapitre central, intitulé «Histoire d'Angus et de Jessica ». Le lecteur ne saisit pas d'emblée le rapport de ce chapitre avec les précédents ; la rupture est d'ailleurs matérialisée par la typographie en italiques. Nous ne citerons que quelques passages choisis, qui donneront un aperçu partiel de ce texte complexe, digressif, émaillé d'allusions à de nombreux intertextes. Il commence en ces termes :

Aussi, la première fois que Miss Peradventure, en sortant de chez elle, lut sur le mur d'en face l'inscription suivante, tracée avec une craie de feu :

JESSICA, VOUS M'AVEZ
COMBLE D'AMOUR

- elle ne douta pas un instant que cela s'adressât à elle, encore que son prénom fût Primrose. Mais est-il possible qu'on soit à jamais fixée par le petit nom que des parents vous choisissent, quand il suffit d'un homme pour vous faire changer le grand ? [...] la jeune fille devint Jessica rien qu'à regarder le mur d'en face, où de vieilles affiches déchirées rêvaient encore à des savons de transparence ou des thés couleur de lune. Et se mit à imaginer Angus Gillravager.

Personne ne peut dire si elle commença par son nom ou par son physique. (BO, p. 300-301)

7 Dans ce chapitre qui appartient visiblement au genre de la rencontre amoureuse, le récit n'installe qu'un seul personnage réel: Primrose Peradventure, qui, s'imaginant destinatrice d'une inscription, adopte un autre prénom, Jessica. Le deuxième protagoniste du couple amoureux, Angus Gillravager, est imaginaire. Suit un assez long récit de la journée folle de cet Angus, récit que l'on peut sans certitude supposer issu de l'imagination de Primrose. La rencontre amoureuse y est retardée par une suite de rendez-vous manqués. Ce n'est qu'au matin que la rencontre a lieu :

Puis, s'étant soudain rappelé Jessica, la tête en feu, [Angus] s'habilla dans son costume de vitrier afin d'être méconnaissable et courut d'une traite, au risque de casser ses vitres dans l'escalier, jusqu'à la place de Peradventure et Peradventure (... 
). Or, ouvrant la porte au-dessus de laquelle on pouvait lire, dans la pierre taillée, un splendide 1818 d'époque, Miss Peradventure s'avança sur la place, regarda le vitrier courbé sous sa charge de monocles géants, et s'écria avant d'y avoir pensé : Angus ! à moins que ce n'eût été Gillravager qui le premier se vit déchiré, du ventre à la bouche, d'un Jessica! si formidable, que sur la Tamise au loin, les remorqueurs y répondirent par le barrissement des éléphants à la fontaine (vous voyez ce que je veux dire). Et, comme il lui ouvrait tout grand les bras de sa jeunesse, elle y précipita tout le jaune pâle de ses fleurs. (BO, p. 311)

Le dénouement, on le voit, remplit finalement le contrat générique : on a bien une scène magnifique de coup de foudre ; celle-ci n'en est pas moins surprenante, au regard de ce que le texte nous a donné à savoir des deux protagonistes amoureux. Comment le personnage d'Angus, né de l'imagination de Jessica, pourrait-il finalement la prendre dans ses bras? On peut toujours supposer que son rêve a fini par donner consistance réelle au jeune homme: ceci ne serait pas insurmontable dans le cadre d'un récit qui emprunte plusieurs éléments au merveilleux... Mais les choses deviennent vraiment insolubles avec le commentaire du narrateur, une page plus loin :

Mais ici se pose une question: qui donc, de Primrose Peradventure ou de Gamy

John, le photographe de Chelsea, lequel ne s'appelait pas plus Angus qu'elle Jessica,

qui donc, qui donc inventa l'autre? (BO, p. 312)

Le lecteur est contraint de revenir sur sa première interprétation du conte : Angus n'a pas pris corps à partir de l'imagination de Primrose ; il existait indépendamment d'elle, bien que sous un autre nom. Deux personnages réels, nommés Primrose et Gamy John, se rencontrent donc; avant cette rencontre, Gamy John connaissait l'apparence de Primrose, mais pas son nom, qu'il a inventé. Elle, de son côté, ne connaissait ni le nom de Gamy John, ni son physique: elle a inventé les deux. Et pourtant, ils reconnaissent mutuellement leur apparence et leur nom.

10 Il était cependant impossible que Jessica identifie Gamy John, l'auteur de l'inscription, qu'elle n'avait jamais vu. Il faut donc que l'amoureux soit à la fois Gamy John, personnage réel (puisque le texte nous le dit), et Angus, personnage imaginé par Jessica. C'est là que le récit devient proprement, selon nous, contradictoire: non parce qu'il comporte des éléments impossibles dans notre univers actuel, comme la présence de fées, ou comme le fait qu'un personnage imaginaire prenne corps. Mais parce que son dénouement ne peut s'entendre que comme une contradiction logique : Angus, au même moment du récit, est et n'est pas imaginaire. Il est et n'est pas réel. Il peut et ne peut pas la prendre dans ses bras, au mépris du principe de non-contradiction. L'histoire d'Angus et de Jessica est une histoire logiquement impossible: pas seulement du point de vue de ce qui est matériellement possible dans notre réel, mais du point de vue de ce que la raison humaine peut logiquement concevoir.

11 En termes narratologiques, la contradiction prend ici la forme particulière de ce que G. Genette a appelé la "métalepse narrative », définie comme franchissement entre niveaux narratologiques étanches, c'est-à-dire «toute intrusion du narrateur ou du narrataire extradiégétique dans l'univers diégétique (ou de personnages diégétiques dans un univers métadiégétique, etc.), ou inversement ${ }^{6} »$; ici le personnage du récit intérieur imaginé par Primrose passe dans le niveau de sa narratrice. Mais la difficulté logique de la métalepse simple est redoublée par la question: "qui donc inventa l'autre ${ }^{7}$ ?». S'ajoute alors au problème de la contradiction une impossibilité logique vertigineuse, celle que l'on appelle aujourd'hui "boucle étrange»-depuis le fameux Gödel, Escher, Bach de Douglas Hofstadter : il s'agit d'une boucle qui revient rétroactivement à son point de départ, tout 
en changeant de niveau en cours de route. L'un des exemples qu'en donne Hofstadter est cette célèbre gravure d'Escher :

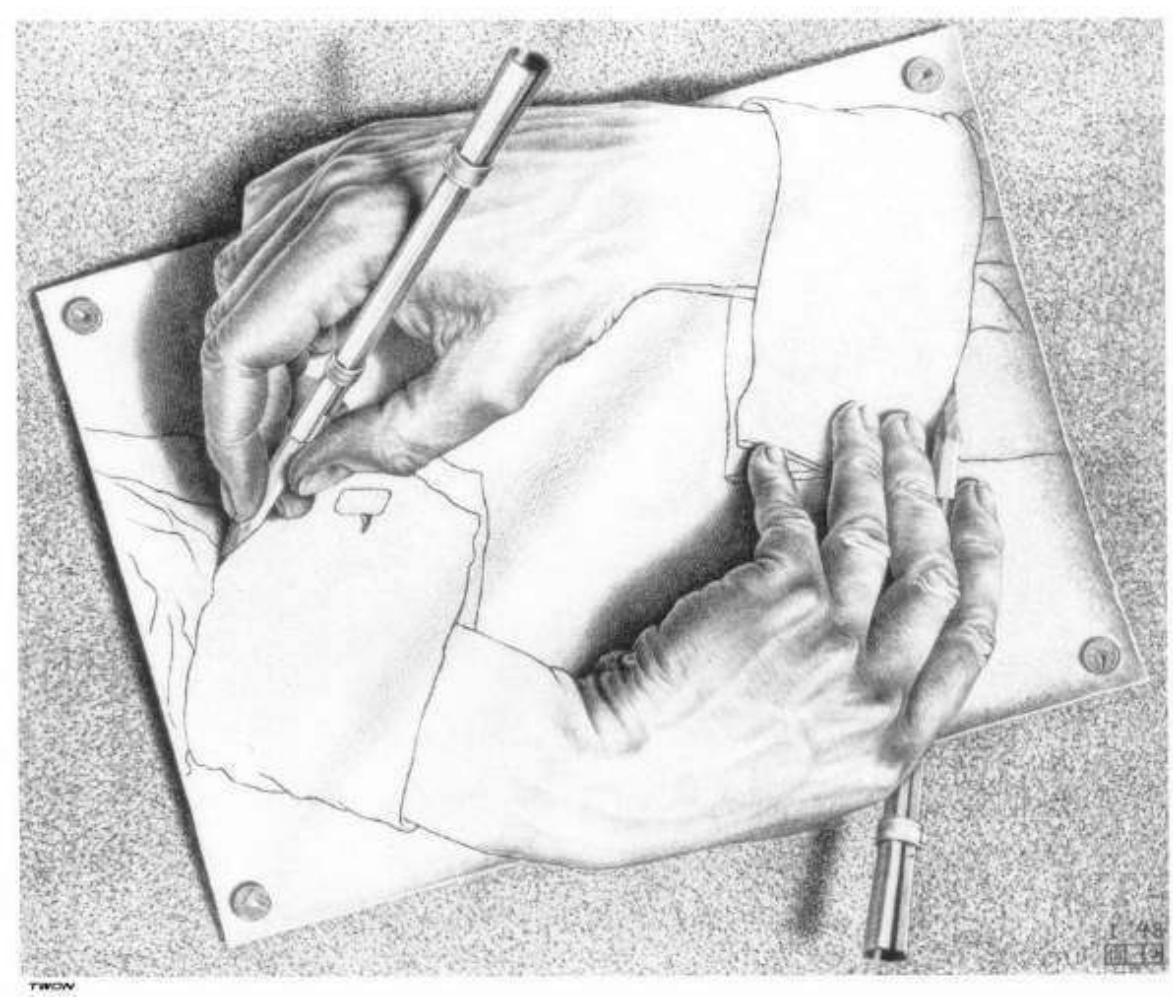

M.C. Escher, « Dessiner », 1948

12 Ici, dit Hofstadter, « le déplacement abstrait d'un niveau à un autre serait le saut vers le haut de dessiné à dessinant (ou, de manière équivalente, d'image à artiste), ce dernier niveau étant intuitivement " au-dessus » du premier $^{8}$ ». Le niveau de la représentation inclut et est inclus dans le niveau du représenté, boucle logiquement impossible en ce qu'elle est à la fois circulaire et transgressive. Une main dessine une main et est simultanément dessinée par cette dernière... Le «qui inventa l'autre » aragonien en est une formulation très proche : Angus imagine Jessica qui simultanément imagine Angus.

Comment interpréter la contradiction logique dans le récit aragonien, à défaut de la lever? Plusieurs pistes interprétatives, plus ou moins apparentes, sont mobilisables : la théorie de l'invention réciproque est donnée au début du chapitre comme exemplification de l'amour fou («les amants sont imagination de l'un par l'autre», p. 300) et permet à Aragon de se situer à la confluence de deux esthétiques: on sent l'influence de Breton dans cette rencontre magique, pleine de gitanes et de fées; mais la boucle étrange est aussi une réalisation hyperbolique de la théorie du «mentir-vrai » qui infléchit le réalisme aragonien de ces années-là', et qui pose que le réel est toujours invention, et nous invente réciproquement.

Mais le récit contradictoire dépasse les motivations individuelles; ce texte mais aussi, on le verra, plusieurs autres de la même période, sont à mettre en regard avec un ensemble de données culturelles qui, à partir du milieu des années 1960, favorise un intérêt renouvelé pour la contradiction logique. 


\section{Au fondement du récit contradictoire moderne : politique, philosophie, sciences des textes}

15 Le récit aragonien date de 1967 ; l'exploitation de la contradiction qui le constitue n'est pas nouvelle en littérature. Pour ne citer que deux auteurs en rapport direct avec notre texte, Aragon a lu et même traduit Lewis Carroll ${ }^{10}$, et il apprécie son goût tout mathématicien du paradoxe logique; il connaissait aussi Allais qui, comme nous le verrons tout à l'heure, cultivait ce type de contradictions. Toutefois, si la potentialité du récit contradictoire est déjà ouverte, il semble que la littérature ne se saisit pleinement des modalités de contestation du principe de non-contradiction qu'à partir de la fin des années 1950. Dès les années 1950, l'âge d'or de la science-fiction voit déjà naître un certain nombre de textes représentatifs, comme Le Monde des $\bar{A}$ (monde des non-A), qui imagine une société régie par une logique non-aristotélicienne ${ }^{11}$. Les années 1960 voient l'intérêt pour la contradiction s'implanter dans des genres plus traditionnels. En 1963, est traduite la nouvelle de Cortazar "Continuité des $\operatorname{parcs}^{12}$ ", où le lecteur d'un livre est assassiné par l'un de ses personnages; en 1965, le principe de la boucle étrange est magistralement illustré par Queneau dans Les Fleurs bleues ${ }^{13}$, où Cidrolin rêve du Duc d'Auge qui rêve de Cidrolin. Les années 1970 verront éclore la boucle étrange dans de nombreux récits, entre autres dans la mouvance du Nouveau Roman, tels Triptyque de Claude Simon (1973), Projet pour une révolution à New York (1970) ou Topologie d'une cité fantôme (1976) de Robbe-Grillet...

16 Or, la fortune du récit contradictoire prend particulièrement sens, durant ces décennies, si on la rapporte à plusieurs déterminations esthétiques et culturelles. Leur mention nous paraît à présent nécessaire, tout genre ou sous-genre étant déterminé par un air du temps qui excède le discours littéraire ; nous insisterons sur trois d'entre elles.

\section{Contradiction et marxisme}

17 Cette détermination concerne certainement Aragon, et elle permet de motiver son goût $\mathrm{du}$ non-sens et de la contradiction. On sait que dans la logique aristotélicienne, la contradiction se situe dans la pensée, où elle est le signe d'un argument fautif. Le raisonnement droit obéit aux trois lois de la logique, loi d'identité, loi de noncontradiction et loi du tiers-exclu. Mais à partir du XIXe, des philosophes comme Fichte, Schelling ou Hegel notamment s'intéressent au contraire à la valeur positive de la contradiction, qui permet de penser des paradoxes comme celui du mouvement. Marx reprend alors à son compte le dépassement de la non-contradiction par l'idéalisme allemand, et le pousse à son terme ultime en concevant la contradiction comme essence et non comme apparence : « L'erreur principale de Hegel est de concevoir la contradiction apparente comme unité de l'essence, dans l'Idée, alors que cette contradiction est dans son essence plus profonde, c'est une contradiction essentielle ${ }^{14} »$. On comprend ainsi pourquoi la contradiction chez Lewis Caroll prenait pour Aragon une valeur toute contestataire ${ }^{15} \ldots$

18 L'attention à la contradiction a sans doute été renforcée chez lui par un relais d'importance en France, celui de Frédéric Paulhan. Ce philosophe touche-à-tout, mentionné dans Blanche ou l'oubli (p. 352), publie en 1919 un opus intitulé La Logique de la contradiction. L'ouvrage, qui fait de la notion un usage plus lâche que la dialectique 
hégélienne ou marxiste, valorise son rôle créateur : «certains usages de la pensée, ceux où domine l'exercice de l'imagination, supposent continuellement la contradiction et lui doivent leur gloire ». Même dans les sciences dures, il faut selon Paulhan appeler à une « casuistique logique ${ }^{16}$ » qui admettrait la contradiction sans y voir à tout prix la trace d'un raisonnement fautif.

\section{La contradiction dans les sciences exactes}

La revendication de Paulhan annonce la révolution qui va avoir lieu dans les sciences à une décennie de là. Les Principia mathematica de Russel et Whitehead, publiés entre 1910 et 1913 consistaient en l'élaboration de règles logiques propres à éradiquer toute contradiction dans la théorie des ensembles ${ }^{17}$, règles qui interdisent notamment à un ensemble de se contenir lui-même. Or, en 1931, le théorème de Gödel remet en question toute entreprise de ce type, en montrant que le caractère non-contradictoire de quelque système axiomatique que ce soit est impossible.

De façon concomittante, le développement de la physique quantique, notamment entre 1930 et 1950, apporte une révolution conceptuelle d'envergure en physique, en remettant en cause (au niveau subatomique) les catégories kantiennes qui sont celles par lesquelles nous appréhendons le monde : ainsi la non-localité des particules quantiques ou le fait que l'observation est indissociable du système observé, pour ne citer qu'eux, posent un véritable défi à la raison.

21 Il est naturellement vain de penser qu'Aragon ou d'autres aient pu prendre connaissance du théorème de Gödel dans les années 1930 ou des théorisations quantiques dans les décennies suivantes. Toutefois la révolution épistémologique induite par ces deux théories a été d'une telle ampleur qu'elle comporte des répercussions dans tous les domaines de la pensée. La philosophie, la psychanalyse lacanienne, la sémiotique et les sciences du texte en général - sans parler de la littérature, notamment de science fiction - s'en saisissent à partir des années 1960 et leur influence est certaine sur la création littéraire, et notamment sur le goût de la contradiction qui l'anime à partir des années 1960.

\section{Logos, contradiction et sciences des textes}

Un autre mouvement de pensée est concomitant (et peut-être en partie liée) à ces renouvellements de la raison scientifique. Il s'agit de la remise en cause, en sémiotique et en critique littéraire, de la valeur jusque-là attachée au logos en tant que mixte de langage et de raison, et garant du raisonnement juste. Dans la conception aristotélicienne qui domine la rhétorique, et partant les catégories d'appréhension du texte, le logos (terme extensif qui désigne à la fois « discours, langue, parole, rationalité, intelligence... ») fonde à la fois l'homme en tant qu'homme et l'impossibilité de la contradiction. Le principe de non-contradiction est à la fois universel et nécessaire, dans la réalité comme dans le logos, puisque « les énoncés opposés ne sont pas vrais simultanément ${ }^{18}$ ». L'homme fait toujours usage d'un discours sensé, sans cela il n'est pas un homme ; et par là, « le principe de noncontradiction se prouve et s'instancie en cela seul qu'il est impossible qu'un mot ait et n'ait pas le même sens ", selon l'analyse de Barbara Cassin ${ }^{19}$. Or, nous avons tenté de montrer ailleurs ${ }^{20}$ que la dimension potentiellement exclusive et violente d'une conception ultra-rationnelle du logos commence à être dénoncée à partir de la fin des 
années 1960. Alors que la lignée argumentative et rhétorique continue, encore majoritairement aujourd'hui, à créditer le logos d'un pouvoir régulateur et consensuel ${ }^{21}$, une lignée philosophique qui part de Nietzsche et qui se poursuit jusqu'à Levinas ${ }^{22}$ et surtout Derrida ${ }^{23}$ tend à faire du discours rationnel, fondé sur la non-contradiction, un facteur intrinsèque de violence. Cette remise en cause critique entre dans le champ de la sémiotique et par là dans l'ensemble des sciences du texte à partir de la célèbre leçon inaugurale de Roland Barthes au Collège de France, en 1977, où figure cette formule outrancière, mainte fois commentée depuis : «La langue, comme performance de tout langage, n'est ni réactionnaire, ni progressiste; elle est tout simplement : fasciste ; car le fascisme, ce n'est pas d'empêcher de dire, c'est d'obliger à dire ${ }^{24} »$. On ne peut s'empêcher de voir dans cette critique un effet de la théorie aristotélicienne du logos, qui interdit de dire la contradiction et oblige à poser des énoncés bien formés... La tâche de la littérature est, selon Barthes, de lutter contre cette violence originelle de la langue. A la fin des années 1970, ce programme semble déjà largement en marche ; du moins la vocation noncontradictoire du logos rationnel a-t-elle été largement battue en brèche par la publication de plusieurs récits contradictoires, tels celui d'Aragon et ou ceux de plusieurs Nouveaux Romanciers.

Cette convergence culturelle modifie profondément les données du récit contradictoire, qui présente de nouvelles valeurs interprétatives, et qui ne mobilise pas les mêmes procédés de mise en discours de la contradiction. Nous voudrions maintenant donner un aperçu de ces différences formelles et interprétatives en proposant de dégager deux modèles possibles de récit contradictoire.

\section{Linguistique de la contradiction}

Mettre en discours la contradiction est en soi une gageure : comment plier le logos à l'expression de ce qui lui est si essentiellement hétérogène? La réalisation de la contradiction dans le récit suppose forcément un usage non standard du discours, en tout cas tel que la domination du paradigme logique nous fait habituellement concevoir ce dernier. Dans les cadres de la linguistique textuelle, un récit contradictoire contrevient ainsi aux méta-règles qui président à l'établissement d'un texte bien formé ${ }^{25}$, au premier rang desquelles figure précisément la règle de non-contradiction qui stipule qu'un texte cohérent «n'introduise aucun élément sémantique contredisant un contenu posé ou présupposé par une occurrence antérieure ${ }^{26} \%$.

Selon nous, il est possible de dégager deux procédés formels propres à déroger à cette métarègle, et partant deux modèles linguistiques de récit contradictoire. Pour les exposer avec texte à l'appui, nous ferons un détour par un cours texte d'Alphonse Allais, avant d'en revenir à Blanche ou l'oubli.

\section{Le modèle inférentiel}

Nous évoquerons les propriétés de ce modèle à partir d'un exemple, celui d'« Un Bal bien parisien ${ }^{27}$ ", une nouvelle d'Alphonse Allais datée de 1890 ; nous n'en présentons ici qu'une vision synthétique. La nouvelle, qui précède historiquement le paysage culturel que nous venons d'évoquer, repose pourtant sur une contradiction proche de celle qui nous occupe chez Aragon: Raoul et Marguerite, époux aussi jaloux l'un que l'autre, reçoivent chacun de leur côté une lettre anonyme dénonçant leur moitié respective. 
Selon la première missive, Raoul se rendra au Bal des Incohérents déguisé en Templier, et en galante compagnie. Selon la deuxième, Marguerite ira au même Bal déguisée en Pirogue et non moins galamment accompagnée. Le soir du Bal, «un Templier fin de siècle » entraîne « une Pirogue congolaise » à l'écart et la démasque soudain, avant de dévoiler son propre visage. "Tous les deux poussèrent, en même temps, un cri de stupeur, en ne se reconnaissant ni l'un ni l'autre. Lui, ce n'était pas Raoul. Elle, ce n'était pas Marguerite. » L'aventure sert de leçon aux époux, qui vivent depuis en bonne intelligence.

Le paradoxe plonge le lecteur dans un abîme de perplexité au moins aussi profond que celui où nous poussent Angus et Jessica : il semble que dans la scène finale, le Templier est tout de même Raoul (sans quoi comment expliquer le fait que les époux tirent leçon de l'aventure ?), et de même pour Marguerite... mais le texte nous dit le contraire. A quel moment, et par quel procédé, s'est-on joué de nous? Dans ce récit, la contradiction est linguistiquement construite par une perversion de l'inférence. F. Corblin a montré qu'au moment de la description du Bal, l'expression indéfinie un Templier est spontanément intégrée par le lecteur dans la « chaîne de référence ${ }^{28}$ » des expressions référentielles qui désignent Raoul, quand bien même ce raccordement n'était pas linguistiquement nécessaire : l'indéfini a normalement vocation à présenter un individu qui n'a pas été mentionné préalablement... Mais le lecteur est poussé à ce raccordement fautif par une exigence de cohérence supérieure, qui exige la mise en relation des lettres et de l'épisode du Bal.

C'est donc sur les inférences construites par le lecteur que repose la contradiction, et non, à proprement parler, sur le texte lui-même : la meilleure preuve en est qu'il existe des interprétations non-contradictoires de ce texte ${ }^{29}$. La contradiction peut se cantonner à l'apparence: elle n'est pas dans l'être, ni dans le langage, elle n'apparaît que dans l'activité interprétative. Il s'agit là selon nous d'un modèle a minima du récit contradictoire - ce qui n'enlève naturellement rien à sa force paradoxale, ni à sa drôlerie.

\section{Le linguistic turn du récit contradictoire : désignation propriale, deixis et métalepse}

Tout autre est le deuxième modèle linguistique de récit contradictoire. Celui-ci prend pleinement en compte les révolutions philosophiques, politiques, scientifiques et culturelles que nous avons évoquées dans le deuxième temps de notre réflexion: il introduit la contradiction dans le fonctionnement du logos lui-même, en pervertissant les mécanismes des expressions référentielles. L'« Histoire d'Angus et Jessica » appartient à ce deuxième « moment » du récit contradictoire.

Dans le texte aragonien, ce ne sont plus les mécanismes inférentiels de l'interprétation qui sont faussés, mais les procédés référentiels de la nomination. En effet, la double nature contradictoire des personnages (qui sont à la fois réels et imaginaires, inventés et inventeurs de l'autre), est réalisée par un procédé majeur : la duplicité de Primrose est signifiée par sa désignation par deux prénoms, indifféremment utilisés l'un pour l'autre. Quant à la double nature d'Angus, elle est posée par le raccordement explicite entre les noms propres « Angus » (inventé) et « Gamy John » (réel).

31 C'est la connaissance approfondie qu'avait Aragon des débats linguistiques de son temps qui lui permet de situer la contradiction au cœur de la référence linguistique, selon deux 
procédés : il se fonde sur la théorie du nom propre (dorénavant NP) pour en pervertir l'usage; et il transfère l'incertitude référentielle qui en résulte aux expressions déictiques, disséminant par là la « boucle étrange » à tous les niveaux narratifs.

\section{Pervertissement de la référence propriale}

(i) Aragon travaille deux traits définitoires du nom propre dans toute une tradition linguistique : sa vacuité sémantique et son unicité référentielle.

La théorie de J.S. Mill, qui définit le NP comme vide de sens ${ }^{30}$, est reprise de Kripke à Saussure, de sorte qu'Aragon la connaît très certainement. Or Aragon, dans son court récit, fait constamment entorse à la non-signification du nom, par le biais de l'antonomase; ses NP sont originellement des noms communs, c'est-à-dire des noms pourvus d'un sens lexical descriptif: ainsi Peradventure "signifie la possibilité pour une chose d'être ainsi ou autrement» (BO, p. 301), et Gillravager est «est un mot d'Ecosse qui a vieilli, on l'employait du temps de Keats, nous dirions chahuteur » (BO, p. 305). Or, dans la théorie millienne, la non-signification du nom est la condition de son unicité ; c'est parce que le nom ne signifie pas qu'il ne désigne qu'un seul être. Avec la signification du NP, Aragon se donne donc le moyen de remettre en question son unicité référentielle.

Celle-ci est une deuxième caractéristique essentielle du NP, formulée dès la tradition logique de Port-Royal, chez Beauzée: «Les noms propres sont ceux qui désignent les êtres par l'idée singulière d'une nature individuelle ${ }^{31} »$. Ce trait discriminant est repris avec insistance chez Damourette et Pichon, dans le chapitre VII du premier livre de Des mots à la pensée qu'Aragon avait lu de près - Pichon est même un personnage à part entière de Blanche ou l'oubli32. Dans cette tradition, il existe une relation bijective entre le NP et son porteur : à chaque NP un référent unique, à chaque être un seul NP.

Or, c'est précisément en pervertissant cette relation bijective entre le nom et son porteur qu'Aragon rend possible la contradiction référentielle. Le nom de Jessica, dans le récit, a deux porteurs : dans la digression féérique, l'une des fées prend l'apparence de Jessica, et est nommée ainsi par Angus. Inversement, chaque protagoniste porte deux noms, on l'a $\mathrm{vu}$ : la duplicité dénominative signe la double nature contradictoire de chacun.

(ii) Enfin, Aragon exploite l'un des aspects les plus novateurs du chapitre VII de l'ouvrage de Damourette et Pichon, la modification du nom. Précurseurs de ce point de vue ${ }^{33}$, les auteurs s'y intéressent à des exemples comme «Une Carthage neuve est sur le point de surgir ", où le nom propre est accompagné d'un article et un adjectif. Leur analyse est la suivante : c'est un cas « dans lequel sans que l'extension sémantique du nom propre soit aucunement changée, il est distingué, à l'intérieur même de sa personnalité, plusieurs espèces, en raison des points de vue divers auxquels on peut l'envisager ${ }^{34}$ ».

Il y a plusieurs exemples de noms propres modifiés dans l'«Histoire d'Angus et de Jessica » : « la nouvelle Jessica eût pu partir des genoux du jeune homme » (BO, p. 301), « Angus avait beau surveiller la maison des marchands de porcelaine, il n'était jamais là quand sa Jessica sortait » (BO, p. 304); «De toute façon, je ne vous le décrirai pas, ce garçon [Angus], crainte qu'il soit moins beau que le vôtre, dans l'extravagance de son âge et le vacarme de son nom» (BO, p. 301 ; nous soulignons). Tous témoignent précisément de cette "division de l'être en plusieurs espèces ", selon les multiples points de vue, éventuellement contradictoires, sous lesquels on les considère.

Le travail aragonien sur la référence propriale consiste finalement à dévoyer deux des caractéristiques linguistiques du NP (l'insignifiance et l'unicité), et à en exploiter une 
troisième dans le même temps (la modification du nom). C'est ainsi qu'il assouplit et multiplie la référence du nom, qui devient le procédé majeur de la contradiction logique.

\section{Fluctuation de la deixis et métalepse narrative}

39 Le NP n'est pas la seule expression référentielle touchée; la fluctuation de la référence atteint une autre catégorie, celle des pronoms de l'interlocution, les déictiques je et tu. Ce roman extrêmement complexe et foisonnant, qui narre globalement les errances de Geoffroy Gaiffier, un linguiste qui peine à surmonter le départ de sa femme Blanche survenu dix-huit ans auparavant, matérialise l'errance d'un je privé de son tu amoureux par la mise en doute systématique de la référence des déictiques :

J'ai essayé de ne plus être celui qui parle, comme on dit, à la première personne, de ne plus être la première personne. Parce que cela me faisait mal où j'étais amputé de toi. Atrocement mal. La première personne, d'être la première, en suppose une seconde, la seconde. Je saignais de dire encore je... d'être ce je qui ne s'adresse plus à personne (p. 390).

40 Dans le tu directement appelé par le je, on retrouve l'influence visible de Benveniste, qui est même cité deux pages plus loin. Geoffroy délègue à plusieurs reprises sa voix narratoriale ; le je se fait extrêmement fluctuant, le nouveau narrateur du récit étant peu identifiable et vraisemblablement pluriel $^{35}$.

41 L'incertitude et la multiplication contre-nature de la référence propriale s'étend donc à la référence déictique. Or, références déictique et propriale ont en linguistique bien des points communs, à commencer par le fait qu'elles sont toutes deux non descriptives. Après la grammaire de Port-Royal, c'est justement Benveniste, qu'Aragon connaissait bien, qui a mis en évidence les ressemblances entre déictiques (toniques) et noms propres : "À la ressemblance et à la différence du nom propre social, MOI est, dans l'instance de discours, la désignation autique de celui qui parle : c'est son nom propre de locuteur ${ }^{36} »$. Pour le dire dans les termes de Mill, le je est vide de sens, tout comme le NP. D'autre part, le je suppose également l'unicité référentielle. On voit que comme le porteur $\mathrm{du} \mathrm{NP}$, le locuteur se multiplie et devient apte à prendre plusieurs visages.

Or, démultiplier le je est une expérimentation plus pernicieuse encore que dédoubler les porteurs des noms propres. En effet, la perversion de la référence déictique sape tout l'édifice de l'origine narratoriale unique. Le je non situable peut dès lors remonter et descendre les niveaux narratologiques, et créer des métalepses narratives multiples qui seront autant de défis à la règle de non-contradiction.

C'est bien ce qui se passe dans le roman d'Aragon. Pour détailler la dissémination de la contradiction linguistique au cœur de toutes les strates énonciatives, il nous faut en dire un peu plus sur la structure narrative dans laquelle prend place l'Histoire d'Angus et Jessica. Pour présenter de façon très lacunaire l'intrigue complexe et foisonnante de Blanche, on dira que le narrateur Gaiffier, incapable de reconstituer ce que pouvait penser Blanche dans les années qui précèdent leur rupture, fait l'hypothèse d'un personnage fictif, une jeune fille nommée Marie-Noire, chargée de reconstituer pour lui les pensées et activités qui ont mené Blanche à la rupture, et notamment son activité d'écriture. A la fin du chapitre narrant l'histoire d'Angus et Jessica, on comprend que ce récit, raconté à Gaiffier par Marie-Noire, est donné par cette dernière comme le roman que Blanche écrivait avant de quitter Gaiffier... Mais ce dernier conteste l'attribution d'une écriture aussi illogique à Blanche; c'est alors que Marie-Noire la place face à sa responsabilité : si ce n'est pas Blanche qui a imaginé cette histoire, dit-elle, «il faudra en attribuer 
l'enfantement ou à moi-même, ou à vous, Geoffroy, si je ne suis qu'un fantôme de votre propre fantaisie » (BO, p. 315). Sommé d'assumer le récit contradictoire, Gaiffier laisse la parole à un narrateur autre, qui résume le dilemme impossible qui devient le sien : « ou Marie-Noire n'a de réalité que dans l'esprit de Geoffroy Gaiffier, elle est une image de sa rêverie, (...) ou c'est Gaiffier lui-même qui sort de la tête pensante de Marie-Noire réelle » (p. 316). La question «qui des deux inventa l'autre ", ressurgit; la fuite du je déplace la boucle étrange d'un cran. Elle touche le niveau supérieur de la narration (puisque Gaiffier est le narrateur de la plus grande partie du roman), dans une nouvelle métalepse narrative qui ajoute au vertige textuel. Et la métalepse finit, à la fin du chapitre, par englober tout processus narratorial possible, lorsque le nouveau narrateur prétend à la page suivante : «n'était-ce pas moi, devant sa porte, qui écrivis (...) Jessica, vous m'avez comblé d'amour » (p. 317). Dans l'interchangeabilité du je, n'importe qui peut prendre la place de tout actant, à quelque niveau que ce soit. Il faudrait décidément imaginer une sémiologie à la Escher pour tenter de donner une représentation visuelle de ces niveaux énonciatifs aux hiérarchies réciproquement enchevêtrés...

Chez Allais, c'était les chaînes référentielles établies par les inférences du lecteur qui fondaient la contradiction; chez Aragon, ce sont les expressions référentielles en ellesmêmes qui disséminent la contradiction à toutes les strates narratives. Dans ce modèle linguistique a maxima, l'impossibilité logique quitte le lecteur et son activité interprétative, pour habiter la langue - ce qui est sans doute moins moqueur, mais bien plus radical.

Voici donc deux modèles possibles de deux récits contradictoires: modèle inférentiel contre modèle référentiel, modèle logique contre modèle linguistique. Le deuxième modèle fonctionne, bien plus que le premier, a maxima. C'est que publié en 1967, il se situe pleinement dans le paradigme politique, philosophique et culturel que nous avons retracé dans le deuxième temps de notre réflexion. La contradiction n'y est plus un accident drolatique de l'interprétation; elle entre pleinement dans la langue, dans le processus de sémiotisation et d'appréhension $\mathrm{du}$ monde. Cette contradiction souveraine, sans rémission, fait du logos lui-même son terrain, signant la déconstruction de la logique aristotélicienne - et au-delà, la nécessité de construire une perception, une représentation et une éthique renouvelées.

\section{NOTES}

1. Aristote, Métaphysique, 1005b, 19-20.

2. Postmodernist fiction, London, Methuen, 1987, notamment chapitre 3.

3. Le Nouveau Roman, Seuil, «Points", 1990 [1973], p. 152. Pour une étude approfondie de la notion de discohérence, nous renvoyons à I. Yocaris, «La discohérence dans Triptyque et Leçon de choses de Claude Simon ", Cohérence et discours, F. Calas éd., Paris, PUPS, 2006, p. 399-408.

4. Bibliothèque de l'Entre-Mondes. Guide de lecture, les transfictions, Gallimard, "Folio / SF », 2005 ; "Narratologie thématique et narratologie discursive: le cas des transfictions", Narratologies contemporaines, J. Pier et F. Berthelot dir., Paris, Editions de archives contemporaines, p. 75-89.

5. Blanche ou l'oubli, Paris, Gallimard, 1967, p. 322 (dorénavant BO). 
6. Genette, Figure III, Paris, Seuil, 1972, p. 244.

7. Celle-ci, notons-le, est un peu biaisée, car elle laisse entendre que le récit repose sur une pure symétrie, ce qui n'est pas exact: si Jessica a en effet inventé le nom et l'apparence d'Angus, Angus, lui, n'a inventé que le nom de Jessica, qu'il avait déjà rencontrée et qui est donc réelle à ses yeux ; Jessica, par conséquent, n'est pas totalement imaginaire.

8. D. Hofstadter, Gödel, Escher, Bach. Les Brins d'une Guirlande Éternelle, Paris, Dunod, 1985 [1979], p. 130.

9. Titre d'une nouvelle de 1964 publiée dans les CEuvres romanesques croisées d'Elsa Triolet et Aragon, tome 4, Paris, Robert Laffont, 1964. Selon «L'Après-dire » de Blanche ou l'oubli, le roman serait tiré de cette nouvelle.

10. Aragon traduisit La Chasse au Snark, une agonie en huit crises, 1929, aux éditions The Hours Press ; le nom de Carroll est cité dans Blanche ou l'oubli, p. 185.

11. A. E. van Vogt, Le Monde des Ā, traduit de l'américain par Boris Vian, Hachette et Gallimard, « Le Rayon fantastique », 1953.

12. Julio Cortazar, «Continuidad de los Parques » (1956), Fin d'un jeu, traduit de l'espagnol par C. et R. Caillois, Paris, Gallimard, 1963.

13. Paris, Gallimard, 1965.

14. K. Marx, Critique de la Philosophie Politique de Hegel, Cuvres III, Paris, Gallimard, Bibliothèque de la Pléiade, 1982, p. 973-974. Le capitalisme est ainsi défini comme un système socio-économique fondé sur la contradiction essentielle entre capital et travail, adossée à la propriété privée des moyens de production. Pour une étude détaillée de la notion de contradiction chez Marx, et ses transformations entre l'idéalisme allemand et la dialectique matérialiste, nous renvoyons à Lawrence Wilde, Marx and contradiction, Aldershot, Avebury, 1989

15. Dans un article intitulé « Lewis Carroll en 1931 », Le surréalisme au service de la révolution, $\mathrm{n}$ ๑3, 1931, Aragon rappelle la nécessité de «respecter même le non-sens » (p.26) dans la traduction, celui-ci étant perçu comme un outil de contestation presque révolutionnaire.

16. La Logique de la contradiction, Paris, Felix Alcan, 1911, p. 1, 113 et 132. Deux penseurs polonais, Jan Łukasiewicz et Alfred Korzybski, ont certainement poussé la réflexion plus loin en ce qui concerne l'élaboration d'une sémantique et d'une logique non-aristotélicienne, admettant la contradiction. Mais leurs travaux sont mal connus en France jusqu'aux années 1980, et leur influence dans le champ littéraire nous semble marginale dans ces décennies, du moins en France (ils ont en revanche présidé à l'écriture du Cycle des Ā de van Vogt).

17. En voici un exemple connu sous le nom de «paradoxe de Russel ». La plupart des ensembles ne se contiennent pas eux-mêmes (ainsi, l'ensemble des baleines n'est pas lui-même une baleine): ils sont «quelconques". Mais certains ensembles sont «auto-inclusifs». Soit maintenant $\mathrm{Q}$, l'ensemble de tous les ensembles quelconques; on peut se demander si $\mathrm{Q}$ est un ensemble quelconque ou un ensemble auto-inclusif. Or, la réponse à cette question plonge le logicien dans un abîme de paradoxe. Chaque réponse aboutit en effet à son contraire : si $\mathrm{Q}$ est quelconque, c'est qu'il est auto-inclusif - et inversement...

18. Métaphysique, livre Gamma, $1011 b 13$.

19. Aristote et le logos, Contes de la phénoménologie ordinaire, Paris, PUF, «Bibliothèque du Collège international de philosophie », 1997, p. 20.

20. M. Vallespir et M.-A. Watine, "Le logos est-il violent? Archéologie de la notion », L. KurtsWöste, M. Vallespir et M.-A. Watine dir., La Violence du logos. Entre sciences des texte, philosophie et littérature, Paris, Garnier, à paraître.

21. Voir à ce sujet, par exemple, G. Declercq, "Rhétorique et polémique ", dans La Parole polémique, G. Declercq, M. Murat et J. Dangel (éd.), Paris, Champion, 2003, p. 17-21. La question complexe de la prise en compte, par les théories de l'argumentation d'une dimension violente du discours, notamment polémique, est explorée plus en détail dans M. Vallespir et M.-A. Watine, art.cit. 
22. Notamment dans Totalité et Infini, Paris, Le livre de Poche, 2001, p. 244 et suivantes.

23. «Violence et métaphysique ", L'Écriture et la Différence, Paris, Le Seuil, 1967, p. 117-228.

24. Cuvres complètes, tome 5, Paris, Le Seuil, 2005 [1978], p. 432-433.

25. Il s'agit des méta-règles de répétition, de progression, de relation et de non-contradiction : voir M. Charolles, «Introduction aux problèmes de la cohérence des textes », Langue française, $\mathrm{n}$ - 38, 1978.

26. Art. cit., p. 22.

27. Nouvelle publiée dans Le Chat noir du 26 avril 1890 et reprise dans le recueil A se tordre en 1891.

28. Il s'agit d'une « une suite d'expressions d'un texte entre lesquelles l'interprétation établit une identité de référence" (F. Corblin, Les Formes de reprise dans le discours. Anaphores et chaînes de référence, Presses Universitaires de Rennes, 1995, p. 27). Corblin étudie le texte d'Allais dans un chapitre de cet ouvrage.

29. Nous renvoyons à Corblin pour une présentation d'une de ces solutions, et à notre article pour une seconde.

30. "Les seuls noms qui ne connotent rien sont les noms propres et ceux-ci n'ont, à strictement parler, aucune signification » (J. S. Mill, Système de logique, Bruxelles, Mardaga, 1988 [reprise de l'édition de 1866], p. 35) en est sans doute la formulation la plus connue, même si elle est sans doute biaisée et fautrice de malentendus persistants, comme le montre M.-N. Gary-Prieur, Grammaire du nom propre, Paris, PUF, 1994, p. 15-17.

31. N. Beauzée, Grammaire générale ou exposition raisonnée des éléments nécessaires du langage, pour servir de fondement à l'étude de toutes les langues, Stuttgart-Bad Cannstatt, F. Frommann, 1974 [1767], p. 236.

32. Sur les relations d'Aragon à Damourette et Pichon, et à la linguistique en général, nous renvoyons à Watine, art. cit.

33. Voir à ce sujet S. Leroy et V. Muni Toke, « Une date dans la description linguistique du nom propre : l'Essai de grammaire de la langue française de Damourette et Pichon ", Lalies 27, Paris, ENS Éditions, 2007, notamment p. 51 et suivantes.

34. Op. cit., p. 526.

35. Un passage (parmi beaucoup d'autres du même type) attestera de cette labilité : « Et moi... qui ne me passe pas de romans... qui, moi ? Le moi dont Marie-Noire a besoin pour se passer de moi. Le moi qui crée, aussi bien à le lire qu'à l'écrire, le roman, tour à tout auteur et lecteur, le moi dont le pluriel est ce nous variable (...)» (p. 131).

36. "L'antonyme et le pronom en français moderne », Bulletin de la Société de linguistique de Paris, tome LX, fascicule 1, 1965, repris dans Problèmes de linguistique générale II, Paris, Gallimard, 1974, p. 200.

\section{AUTHOR}

\section{MARIE-ALBANE WATINE}

Maître de conférences en langue française, Université Nice Sophia Antipolis, CNRS, BCL, UMR 7320 